\title{
Identification of material properties from full field measurements of a sound field interacting with a solid
}

\author{
S. Vanlanduit ${ }^{1, \mathrm{a}}$, C. Vuye ${ }^{2}$, R. Longo ${ }^{1}$, and P. Guillaume ${ }^{1}$ \\ ${ }^{1}$ Dept. Mechanical Engineering, Vrije Universiteit Brussel, Pleinlaan 2, B-1050, Brussels, Belgium \\ ${ }^{2}$ Dept. Industrial Science and Technologie, Artesis Hogeschool, Antwerpen, Belgium
}

\section{Motivation}

The identification of the mechanical material properties of orthotropic and porous materials is of primordial importance in many disciplines. In the medical field the identification of the porosity and stiffness of bone material can help in the diagnosis of bone growth and the early detection of anomalies. In structural engineering material identification is a tool to monitor the presence and the extent of damage. In the last decades ultrasound waves have been used with success for material identification purposes in these various disciplines.

For orthotropic or porous materials the interactions of the ultrasound wave with the material should be measured at different angles of incidence. Since usually ultrasound transducers are used in combination with a mechanical traverse system, the measurement time can be quite high when a large number of angles of incidence are to be tested. In this article we present a method to estimate material properties from focused sound fields that contain several incidence angles simultaneously. Furthermore, in order to reduce the measurement time, the (ultra)sound fields are measured using a full field optical measurement technique.

\section{The proposed measurement technique}

In this paper we will use a spherical focused beam emitter to generate an ultrasound beam. When the focal point coincides with the surface of the material, the incident wave on the material contains simultaneous components with different angles of incidence at each instance of time.

The amplitude and phase of the full incident and reflected ultrasound patterns will be measured using a 3D optical measurement technique (based on a scanning laser Doppler vibrometer). In the past this method has been used for the sound absorption identification of porous acoustic materials [1]. A schematic visualization of the setup is shown in Figure 1. A sound field is generated by an ultrasound transducer. The (plain wave or spherical) sound field interacts with the material under test. The field is measured with the aid of a scanning laser Doppler vibrometer. This is done by aiming the laser beam at a rigid block (that does not vibrate). Since the beam intersects with the sound wave, path length differences will be measured. These path length differences can be related to the integration of the density variation along the laser beam.

\footnotetext{
a e-mail : steve.vanlanduit@vub.ac.be
} 


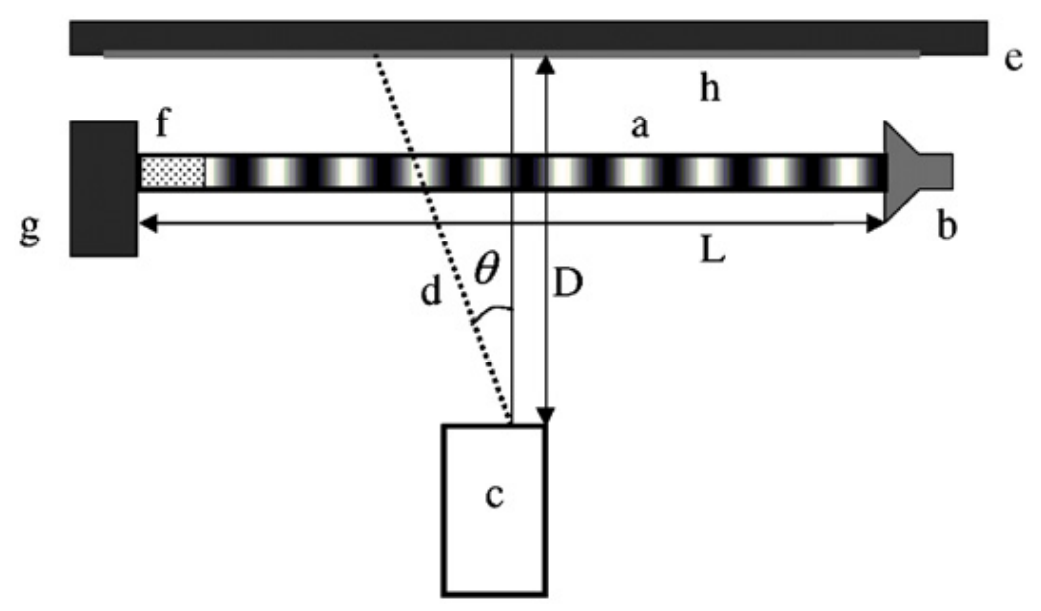

Fig. 1. Experimental setup: (a) (ultra)sound wave, (b) transducer or loudspeaker, (c) Scanning laser Doppler vibrometer (SLDV), (d) laser beam, (e) rigid block, (f) tested material, (g) rigid termination and (h) retro-reflective tape.

\section{The material identification method}

When the spherical sound field in the vicinity of the material has been measured, the measurements can be processed to estimate the orthotropic mechanical properties. To estimate the absorbed acoustical energy in the material in function of the incidence angle Tamura's method can be used [2]. This method uses a two-dimensional Fourier transform to separate the incident and reflected waves.

Once the absorption coefficients in function of the incidence angle are determined the orthotropic material properties can be calculated. This is similar to what was proposed in the so-called polar scan method [3]. For simple material layouts (e.g. a composite with a small number of plies) an analytical expression for the sound-material transfer can be calculated. For more complicated materials a finite element approach could be used. An inverse model technique is used to estimate the material properties.

\section{References}

1. Vanlanduit, S; Vanherzeele, J; Guillaume, P and De Sitter, G., Absorption measurement of acoustic materials using a scanning laser Doppler vibrometer, Journal Of The Acoustical Society Of America, Volume: 117 Issue: 3 Pages: 1168-1172 Part: 1 Published: MAR 2005.

2. Tamura, M; Spatial Fourier-Transform Method Of Measuring Reflection Coefficients At Oblique-Incidence .1. Theory And Numerical Examples, Journal Of The Acoustical Society Of America, Volume: 88, Issue: 5, Pages: 2259-2264, 1990.

3. Van Dreumel, WHM, Speijer, JL, Polar-Scan, A Non-Destructive Test Method For The Inspection Of Layer Orientation And Stacking Order In Advanced Fiber Composites, Materials Evaluation, Volume: 41, Issue: 9, Pages: 1060-1062, 1983. 